contrast, single-handed practices have been able to benefit from the Small Business Rate Relief Scheme, including a $£ 10,000$ grant, and this has greatly helped them to get through this crisis.

This contrast in fortunes begs one question: is it time for the profession to reconsider its fondness for larger practices and to cherish anew, its rich heritage of small, family practices?

C. Marks, Southampton, UK https://doi.org/10.1038/s41415-020-1930-y

\section{Coping well}

Sir, we read Drs Mijiti and Huojia's letter (Psychosomatic problems BDJ 2020; 228: 738 ) with deep empathy. Japan is gradually moving out of the emergency state but remains vigilant against the second wave of COVID-19. As the authors described, patients with chronic pain like Burning Mouth Syndrome (BMS) are at high risk for depression and anxiety with social isolation a possible risk for developing further psychosomatic symptoms. ${ }^{1}$

As of July 2020, two weeks after the re-opening of Psychosomatic Dentistry Clinic (Dental Hospital, Tokyo Medical and Dental University), we do not observe a growing number of patients with new oral psychosomatic disorders. Also, the pandemic chaos did not necessarily worsen our patients' condition. In contrast, some even told us that they felt better by being released from the stress of commuting and mental fatigue of workplace interpersonal relationships. We suppose here that BMS symptoms might not be simply connected with anxiety and depression, as do some psychiatric conditions. In most cases our BMS patients are coping well with this unusual situation, beyond our expectations.

The activities of our psychosomatic dental services were interrupted and reduced for several months, being considered as non-urgent. Almost all patients had to be followed up with telemedicine, including brief psychological counselling via telephone; continuing the prescriptions (mainly for antidepressants) via fax services, in cooperation with nearby pharmacies. Many patients expressed thanks for keeping up the medication while being able to avoid infection risks such that we suggest online consulting was useful for BMS patients during social distancing. It would also be useful in the event of a second wave of COVID-19 and other natural disasters, like earthquakes or typhoons.

Treating a dental patient with

psychosomatic problems is always difficult. ${ }^{2}$ However, given the many fundamental changes to daily lives in the current pandemic, we suggest expanding telehealth and telemedicine to follow up oral

psychosomatic patients at home. This also aligns with Dr Mijiti's idea of training dental hygienists to apply some psychological techniques of Cognitive Behavioural Therapy (CBT). ${ }^{3}$

\section{T. T. H. Tu, A. Toyofuku, Tokyo, Japan, H. Matsuoka, Hokkaido, Japan}

\section{References}

1. Piraccini $E$, Byrne $H$, Taddei $S$. Chronic pain management in COVID-19 Era. J Clin Anesth 2020; 65: 109852.

2. Toyofuku A. Psychosomatic problems in dentistry. Biopsychosoc Med 2016; 10: 14.

3. Matsuoka H, Chiba I, Sakano Y, Toyofuku A, Abiko Y. Cognitive behavioral therapy for psychosomatic problems in dental settings. Biopsychosoc Med 2017; 11: 18.

https://doi.org/10.1038/s41415-020-1931-x

\section{Temporary filling anaphylaxis}

Sir, a fit and well 33-year-old female presented to A\&E with signs and symptoms in keeping with anaphylaxis. Prior to this manifestation she had placed an 'at home' temporary filling into tooth 35 ; at examination she stated an allergy to cloves and thus we suspected that the material contained eugenol.

After stabilisation in A\&E, she was reviewed by our team. On discussion she decided against removal of the filling under rubber dam or the tooth itself. However, she re-presented approximately 48 hours later with recurrent symptoms of anaphylaxis at which point the tooth was extracted in a theatre setting under local anaesthetic; she made a full recovery.

We would like to highlight this concern due to the increased use of 'at home' filling kits by our patients, as a result of remote triage during the coronavirus pandemic. Here it is important to inform patients to check temporary filling material ingredients and update allergy status when advising such management.

G. Zakary, R. Major, A. Bhaiyat, York, UK https://doi.org/10.1038/s41415-020-1932-9

\section{Judgement and virtual reality}

Sir, dental education is changing as a result of the COVID-19 pandemic and we highlight two aspects of this to affect us as final year students.

We have been informed that dental foundation training (DFT) interviews will no longer be taking place and the recruitment process will be solely placed upon the situational judgement test. This consists of a selection of multiple-choice and ranking-based questions to represent challenging scenarios which dentists may encounter. The news has heightened anxiety levels in many students. As this is the only exam to decide undergraduate ranking among their peers, the amount of pressure placed upon them has been amplified.

Secondly, students in their later years of training are anxious as the lack of clinical exposure inevitably decreases confidence levels. We are fortunate to have experienced hapTEL (haptics in technology-enhanced learning). This award-winning virtual reality technology consists of glasses, a foot pedal and a drill giving students a realistic 3D experience with sound, touch and vision. Benefits include improvements in the positioning of the clinician, enhanced manual dexterity and early adaption to a clinical setting. Precision can be practised as the different layers of the tooth surface can be differentiated, improving tactile sensation. It also serves as a great tool to gain constructive feedback from tutors.

We believe this device would be beneficial to use as an adjunct to fill the gap of lost clinical time and that it should be implemented across other universities to enhance clinical practice to achieve optimum care for our patients.

S. Thakkar, S. Kadia, London, UK https://doi.org/10.1038/s41415-020-1933-8

\section{An opportunity}

Sir, with the increased success of identifying symptomatic COVID-19 cases followed by self-isolation, the main mode of transmission is going to be increasingly through asymptomatic spread. ${ }^{1}$ We are not routinely testing patients who have been selected for an aerosol generating procedure (AGP) and this is based (in Scotland) on the low prevalence of 
infective cases in the community. In fact, the modelling used suggests that 5,000 tests would need to be performed to identify one asymptomatic infective case.

There is an opportunity here to perform a prospective study by testing patients who have been selected for an AGP, to assess this model/hypothesis. Recruiting practices from various geographical locations would be necessary to attempt to catch any local variations but if one or two thousand consecutive cases came back negative, how reassuring that would be?

\section{N. Malden, Kinross, UK}

\section{Reference}

1. Gillespie C. What's the difference between asymptomatic and presymptomatic spread of COVID19? Explore Health 11 June 2020. Available at: https:// www.health.com/condition/infectious-diseases/ coronavirus/asymptomatic-vs-presymptomatic (accessed 12 July 2020).

https://doi.org/10.1038/s41415-020-1934-7

\section{Testing reprise}

Sir, I have just read Dr J. A. Woodcock's letter ${ }^{1}$ in the $B D J$ on testing and find that his proposal has one major flaw. As Prof Jason Leitch has said several times at the Scottish First Minister's briefings, if someone is tested today and the result comes back negative tomorrow, that only proves that they were negative today. It does not prove that they will be negative tomorrow, or on whatever date they may be given an appointment under Dr Woodcock's proposal. Regrettably, I cannot see, therefore, that his suggestion is of any help.

J. Watt, Biggar, UK

\section{Reference}

1. Woodcock J A. Test, test, test! Br Dent J 2020; 228: 902-903.

https://doi.org/10.1038/s41415-020-1935-6

\section{Vertical transmission}

Sir, in connection with the interesting discussion of Richards et al. on coronamolars as a possible congenital disorder of the coronavirus disease (COVID-19), we suggest maternal-foetal transmission (vertical transmission) to predict the coronamolars' development. ${ }^{1}$

Mulberry molars and Hutchinson's teeth are characteristic dental stigmata of children born to mothers with syphilis infection during pregnancy.
Although a recent systematic review has concluded that there is no evidence of the vertical transmission in neonates of mothers with confirmed COVID-19 infection $0.0 \%(0 / 310),{ }^{2}$ the possibility of vertical transmission cannot be completely ruled out due to the few individual neonate cases which were reported positive a few hours after their birth. Vertical infection therefore remains a possibility in the context of COVID-19 thus implying a potential call-to-action for dental researchers and professionals to look for possible orofacial manifestations either in the short- or long-term, once cohorts of congenital COVID-19 are reported.

\section{A. Riad, Brno, Czech Republic, B. Hockova, Banska Brystrica, Slovak Republic}

\section{References}

1. Richards J A, Beaumont I, Beech A N. Coronamolars? Br Dent J 2020; 228: 904

2. Huntley B J F, Huntley E S, Di Mascio D, Chen T, Berghella V, Chauhan S P. Rates of maternal and perinatal mortality and vertical transmission in pregnancies complicated by Severe Acute Respiratory Syndrome Coronavirus 2 (SARS-Co-V-2) infection: a systematic review. Obstet Gynecol 2020; doi:10.1097/ AOG.0000000000004010.

https://doi.org/10.1038/s41415-020-1936-5

\section{Racism}

\section{A global pandemic}

Sir, the tragic death of George Floyd has caused great anguish around the world but, in response, the devaluation and dehumanisation of black people has again come to the fore. This is not an American problem; structural and institutional racism is a problem everywhere around us. Such racism can be overt or covert and it is important to recognise that dentistry is not immune.

Research has shown that dentists' decision making is affected by patient race, with black patients having a greater likelihood of having extractions than white patients. ${ }^{1,2}$ Mistrust of dentists is demonstrated in a study reporting that Black Minority Ethnicity (BME) participants in London believe that, because of their background, they received a poor service, were not respected, listened to or cared about by dentists compared to other non-BME patients. ${ }^{3}$ More generally, within the NHS, ethnic minority patients and clinicians face many injustices. The 2019 Workforce Race Equality Standard (WRES) data report showed that a higher proportion of BME staff experienced harassment, bullying, or abuse from staff compared to white staff. ${ }^{4}$

The $B M J$ have a special issue on racism in medicine which is eye-opening and powerful. It reflects the experiences of doctors and patients from ethnic minority backgrounds. ${ }^{5}$ I believe the $B D J$ should undertake a similar enterprise to encourage more publications on race and racism. Such conversations are needed within dentistry as it is not talked about as much and openly as it should be.

In order to understand this subject, we must look beyond clinical dentistry and appreciate the study of social sciences, philosophy and humanities. It is engraved as a GDC Standard to 'treat patients fairly, as individuals and without discrimination'. It should be embedded within us as humans from the very beginning and we must acknowledge and challenge our own personal prejudices we may have.
I want to stress the importance of acknowledging the structural and institutional racism in dentistry specifically and in society more generally. This is an ongoing issue and we need to not only act and speak up whenever we witness injustice but to engage more in the conversation about race and racism.

K. Villanueva, Hull, UK

\section{References}

1. Patel N, Patel S, Cotti E, Bardini G, Mannocci F Unconscious Racial bias may affect dentists' clinical decisions on tooth restorability: a randomized clinica trial. JDR Clin Trans Res 2019: 4: 19-28.

2. Plessas A. To what extent do patients' racial characteristics affect our clinical decisions? Evid Based Dent 2019: 20: 101-102.

3. Thalassis N. Commissioning World Class Dentistry in Kensington \& Chelsea and Westminster: A race equality impact assessment of how the current approach to the provision of dental services is affecting BME communities, BME Health Forum. 2009.

4. NHS workforce Race Equality Standard 2019 Data Analysis Report for NHS Trusts. Available at: https:// www.england.nhs.uk/wp-content/uploads/2020/01/ wres-2019-data-report.pdf (accessed June 2020).

5. The BMJ. Racism in medicine. Available at: https://www. bmj.com/racism-in-medicine (accessed June 2020). 